\title{
Immune response to gangliosides in a case of Guillain-Barré syndrome after varicella
}

Tetsushi Yoshikawa, Kyoko Suzuki, Sadao Suga, Eizo Miyata, Hiroko Yamamoto, Takeshi Hosokawa, Takuji Kumagai, Yoshizo Asano

\begin{abstract}
An 8 year old girl was admitted to hospital with the typical clinical features of Guillain-Barré syndrome (GBS) after recovering from varicella. Onset of the disease was just two weeks after the onset of varicella in her young sister. Examination of cerebrospinal fluid and nerve conduction studies showed typical findings of GBS. Although serum from both the patient and sister were analysed for autoantibodies to gangliosides and myelin P0 protein, IgM anti-GM1 antibody and antiGD1b antibody were only detected in the patient. HLA DR haplotypes were quite different between the two subjects. This suggests that these autoantibodies may play an important role in the pathogenesis of GBS after varicella zoster virus infection.

(Arch Dis Child 2000;83:172-173)
\end{abstract}

Keywords: Guillain-Barré syndrome; varicella zoster virus; autoantibodies; intravenous immunoglobulin

Guillain-Barré syndrome (GBS), an acute inflammatory demyelinating polyneuropathy, leads to severe quadriparesis and requires mechanical ventilation in about $20 \%$ of patients. Although the pathogenesis of GBS has not been fully elucidated, there has been increasing evidence that points to an autoimmune aetiology. It is considered that preceding infection by various infectious agents may trigger an autoimmune response that causes the disease. There were three reports of GBS caused by varicella zoster virus (VZV) infection before the early 1990s in the English language literature. ${ }^{1-3}$ Although molecular mimicry between a glycoprotein of peripheral nerve myelin and VZV was suggested from the results of sequence searches, ${ }^{4}$ there has been no report discussing autoantibodies to a glycoprotein of peripheral nerve myelin in patients with GBS after varicella. We report here a case of GBS after varicella that was probably transmitted from her sister, and compare the titres of several autoantibodies between the patient and her sister.
Case report

An 8 year old girl presented with typical varicella, two weeks after onset of varicella in her younger sister. Six days after the onset of varicella, she complained of gait disturbance and headache. Three days after onset of the neurological symptoms, she was admitted to our hospital. Following the day of admission, she had considerable muscle weakness, hoarseness of voice, and dysphagia. Deep tendon reflexes were absent in the extremities. Examination of the cerebrospinal fluid showed a slight increase in protein concentrations $(0.78$ $\mathrm{g} / \mathrm{l})$ with no concomitant pleocytosis, and neither myelin basic protein nor oligoclonal IgG bands were detected. Nerve conduction studies showed a reduced motor conduction velocity and a reduced amplitude, suggesting axonal damage. Campylobacter jejuni was not isolated from her stool sample. She was diagnosed with GBS, and an S-sulphonated human immunoglobulin ( $0.4 \mathrm{~g} / \mathrm{kg} /$ day) was administered from the day following admission. Three days after admission, her symptoms deteriorated and respiratory insufficiency developed. She received controlled mechanical ventilation and high dose intravenous gammaglobulin treatment $(1 \mathrm{~g} / \mathrm{kg} /$ day $)$; her respiration quickly improved.

Both the patient and the sister were probably infected with the same VZV strain. Therefore, it was hypothesised that the immune response to VZV in the patient was a trigger for the onset of GBS. To compare the immune response to myelin protein between the patient and sister, we examined autoantibodies (both IgM and IgG class) to monosialoganglioside (GM1, GM2), disialoganglioside (GD1a, GD1b, GQ1b1), trisialoganglioside (GT1b), galactosyl cerebroside (GC), and myelin P0 protein in both the patient (serum obtained nine days after the onset of varicella) and sister (serum obtained at 23 days after onset of varicella). All of these antibodies were measured by enzyme linked immunosorbent assay, as described elsewhere (lower limit of detection in all assays 3.0). ${ }^{5} \operatorname{IgM}$ anti-GM1 antibody (titre 8.7) and IgM antiGD1b antibody (titre 3.3) were detected in the serum of the patient. Additionally serum antiGM1 antibody and anti-GD1b antibody titres were measured in 10 patients with uncomplicated varicella (20 paired samples obtained 
from acute (0-6 days after the onset of varicella) and convalescent (14-20 days after the onset of varicella) period in each case). In no case were antibodies detected (data not shown).

\section{Discussion}

In this patient, both IgM anti-GM1 antibody and $\operatorname{IgM}$ anti-GD1b antibody were detected in serum. To our knowledge, this is the first report showing the titres of autoantibodies in a case with GBS after VZV infection. This result might depend on cross reaction between the two antibodies, because the antibodies react to terminal $\mathrm{Gal}(\beta 1-3) \mathrm{GalNac}$ containing glycolipids. Neither autoantibody was detected in serum obtained from the patient's sister, and 10 other children with varicella. The autoantibodies to gangliosides may play a role in the peripheral neuropathy occurring in the patient. It has been suggested that myelin P0 glycoprotein may be involved in the pathogenesis of GBS. ${ }^{4}$ Significant amino acid sequence homologies have been shown between P0 and the glycoprotein of $\mathrm{VZV}{ }^{4}$ However, antibody to myelin P0 protein was not detected in our patient. Further studies are needed to elucidate the role of autoantibodies to myelin in the pathogenesis of neuropathy following VZV.

We thank Dr Masataka Nishimura (Department of Neurology, National Utano Hospital, Kyoto) for measuring antibody titres to galactocerebrosides. This work was supported in part by grants from Fujita Health University.

1 Sanders EACM, Peters ACB, Gratana JW, Hughes RAC. Guillain-Barré syndrome after varicella-zoster infection. Guillain-Barré syndrome after varicella-zoste

2 Ormerod IE, Cockerell OC. Guillain-Barré syndrome after herpes zoster infection: a report of 2 cases. Eur Neurol 1993;33:156-8.

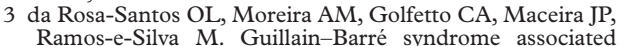
with varicella-zoster infection. Int $\mathcal{f}$ Dermatol 1996;35: 603-4.

4 Adelmann $\mathrm{M}$, Linington C. Molecular mimicry and the autoimmune response to the peripheral nerve myelin P0 glycoprotein. Neurochem Res 1992;17:887-91.

5 Nishimura M, Saida T, Kuroki S, et al. Post-infectious encephalitis with anti-galactocerebroside antibody subsequent to Mycoplasma pneumoniae infection. $\mathcal{F}$ Neurosci 1996;140:91-5. 